\title{
Modeling and experiments of small neuronal networks coupled to micro-electrode arrays
}

\author{
Paolo Massobrio* and Sergio Martinoia
}

\author{
Address: Neuroengineering and Bio-nano Technology Group (NBT), Department of Biophysical and Electronic Engineering (DIBE), University of \\ Genova - Via Opera Pia 11a - 16145 Genova, Italy \\ Email: Paolo Massobrio* - paolo.massobrio@unige.it \\ * Corresponding author
}

from Sixteenth Annual Computational Neuroscience Meeting: CNS*2007

Toronto, Canada. 7-12 July 2007

Published: 6 July 2007

BMC Neuroscience 2007, 8(Suppl 2):P64 doi:10.1 I86/I47|-2202-8-S2-P64

(C) 2007 Massobrio and Martinoia; licensee BioMed Central Ltd.

\section{Introduction}

The use of neuronal cultures coupled to Micro-Electrode Array (MEA) is becoming a widely used and recognized experimental model for studying basic properties of information processing in neuronal systems. On the other hand few models of interconnected neurons are used in conjunction with such devices [1].

In this work, we present a simplified neuronal network made of 60 neurons randomly and synaptically connected. Each neuron is coupled to a microelectrode position, and the network is mapped to reflect the number of recording sites in a MEA. Indeed, the actual number of neurons and connections forming the biological network is much more than those constituting the simplified modeled network. Nevertheless, the simulated architecture is able to account for the neuronal dynamics measured by
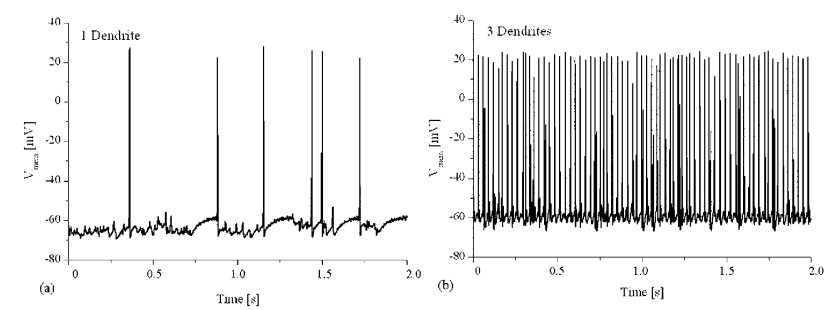

means of the 60 recording channels. Comparing the model with experimental results, it turned out that the overall dynamics of such large networks can be captured by a reduced (small) neuronal network with proper connectivity. In fact, in actual measurements, we are sampling from a small fraction of the neurons constituting the network and it seems that the behavior of such networks can be replicated by few representatives of them. Additionally, our approach can be also conveniently utilized when dealing with low-density patterned networks or interconnected sub-populations [2].

\section{Materials and methods}

All the simulations were carried out by using the software NEURON and the results were compared to the experimental data obtained in our laboratories. Cultured cortical neurons from rat embryos (E18) were plated over
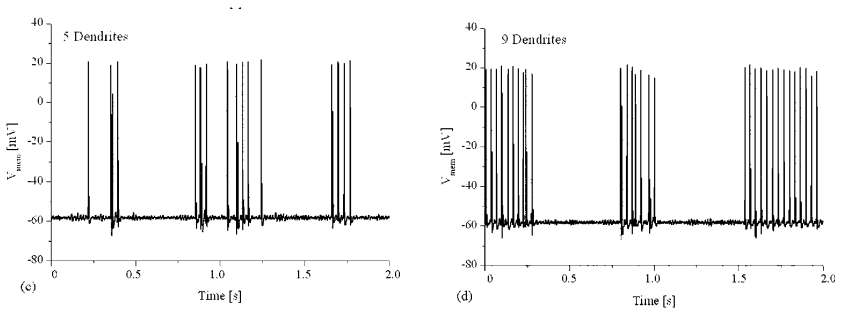

Figure I

NEURON simulations of the spontaneous activity of one neuron of the network as a function of the dendritic arborization. 
MEAs (from Multi Channel Systems, Reutlingen, Germany). The post-processing analysis both for the simulated and experimental data was performed by custom developed software [3].

\section{Results}

We developed a model of a bursting neuronal network by using neurons characterized by Hodgkin-Huxley and passive channels that, in their isolated form, exhibit spiking activity. We showed that by changing the complexity of the dendritic arborization and the degree of connectivity of the network (percentage of inhibitory and excitatory synapses), it is possible to switch from a network spiking activity to a network bursting activity, typical of the mature cortical neuron cultures. The simulated spike trains of one of the sixty neurons of the network, as a function of the morphology of the dendritic tree, are shown in Figure 1. It is evident that in the situation shown in Figure 1a (one dendrite per neuron), a low electrical activity was recorded: that suggests the network activity seems to be ruled by isolated spikes. An increase in the number of dendrites makes the behavior of the network change dramatically: with three dendrites per neuron, the network exhibits tonic activity (Figure 1b) with a high firing rate. At a further increase in the number of dendrites (five or nine), clusters of spikes (bursts) spaced out by silent periods were obtained (Figure 1c and 1d).

\section{Conclusion}

A simple model of a small-scale network coupled to MEAs is presented. Interestingly enough, network bursting behavior using a biophysical neuron model and proper geometry and connectivity is obtained by using spiking neurons. The model is able to capture the overall dynamic of the network with a reduced number of neurons demonstrating uniform spatial behavior and redundancy in such in vitro randomly cultured neuronal systems.

\section{References}

I. Segev R, Shapira Y, Benveniste M, Ben-Jacob E: Observations and modeling of synchronized bursting in two-dimensional neural networks. Phys Rev E 200I, 64: I 1920I-119209.

2. Macis E, Tedesco M, Massobrio P, Raiteri R, Martinoia S: An automated microdrop delivery system for neuronal network patterning on microelectrode arrays. J Neurosci Methods 2007 in press.

3. Vato A, Bonzano L, Chiappalone M, Cicero S, Morabito F, Novellino A, Stillo G: Spike manager: a new tool for spontaneous and evoked neuronal networks activity characterization. Neurocomputing 2004, 58: | I53-1 | 61 .

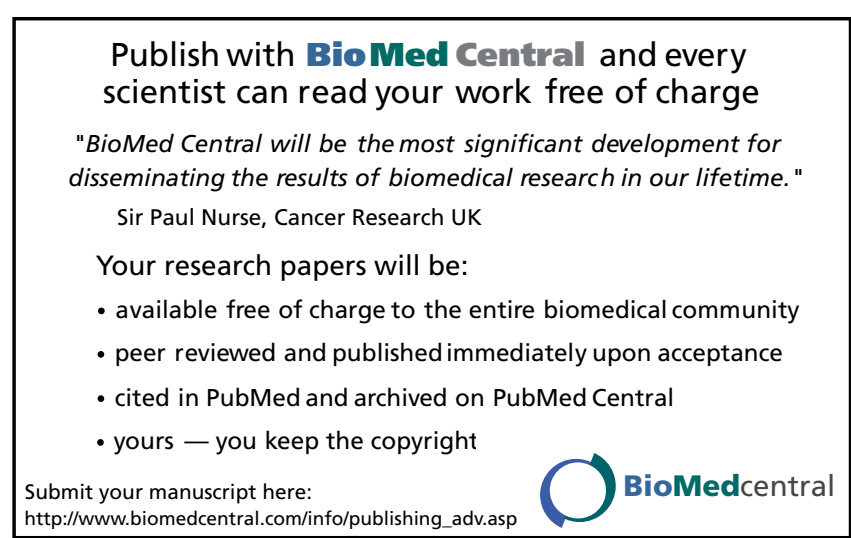

